DE DE GRUYTER

OPEN

G

BULGARIAN ACADEMY OF SCIENCES

CYBERNETICS AND INFORMATION TECHNOLOGIES • Volume 15, No 6

Special Issue on Logistics, Informatics and Service Science

Sofia • 2015

Print ISSN: 1311-9702; Online ISSN: 1314-4081

DOI: 10.1515/cait-2015-0079

\title{
A Study on Geographic Information Services Ontology and Its Applications
}

\author{
Peichao Guan ${ }^{1,3}$, Guobin Zhu'2, Bing Yan ${ }^{3}$ \\ ${ }^{1}$ State Key Laboratory of Information Engineering in Surveying, Mapping and Remote Sensing, \\ Wuhan University, Hubei Wuhan, 430072 China \\ ${ }^{2}$ International School of Software, Wuhan University, Hubei Wuhan, 430072 China \\ ${ }^{3}$ School of Information Engineering, Hubei University of Economics, Hubei Wuhan, 430205 China \\ Emails: guanpc@whu.edu.cn gpc@hbue.edu.cn
}

Abstract: Based on the relevant studies and successful applications of Geographic information ontology and Web services ontology in the last decade, this paper discusses the utility and research trend of the Geographic information services Ontology in the prospective of Geographic information ontology and the Semantization of the Geographic information services. It proposes the concept of Geographic information services ontology and clarifies the purpose of such construction. It further summarizes the procedures and frameworks of the construction in a Geographic information system.

Keywords: Geographic information ontology, Web services ontology, application service, GIS platform.

\section{Introduction}

With the continuous development of information technology and network, geographic information considers their features, such as indigenous and perceptible modes of expression and interactive qualities, making the constructed services possible for frequent and wide use in daily life and manufacturing. At the same time, due to the great quantity of data, variety of formats and heterogeneity of the geographic information services, there are great difficulties in the integration and secondary development. With the help of the development of information sciences and Web Services technology, and the underpinning of the advantageous features (such as package, loose joint and coupling) of the object of its service, geographic 
information services have been in the last decade in transition from a closed, centralized and directional application model to a standardized Web services application model.

In the past few years, a large number of Web service-based geographic information services have been materialized in different platforms and applications. However, this brings forward the question how to render rapid and effective service matching and shared integration in an existing system, based on the individual geographic information services semantics and special requirements of the application. The traditional technology in Web services is based on a grammar, which lacks support of information semantics, resulting in low recall and precision. In order to make the geographic information services mutually operated on a semantic level, and to tackle the problem of heterogeneity in the semantics of geographic information services and the difficulty of integration, relevant studies and attempts on geographic information services ontology are conducted based on the current geographic information ontology and other relevant technologies of semantics. The paper outlines and elaborates the current development of geographic information ontology, expounds the research significance and trends of geographic information services ontology in the perspective of semantization of the geographic information services. It proposes methods and a framework of the application of geographic services ontology based on the current technologies on ontology and further verifies the framework by examples.

\section{Geographic information ontology}

In the perspective of the information sciences, ontology is generally viewed as "a normative description of a certain conceptualized system” [1]. Since 1990-ies, relevant concepts of ontology have gradually been introduced into geosciences. For instance, Nunes [2] first points out that the first step towards establishing a geographic information system is to provide systematic organization and normative description of the geographic entities, their properties and relations. The introduction of ontology to geosciences begins with the study of E g e n h o f e r and M a r k [3]. on geography, which lists ontology as a top priority of all related studies in order to build a popularized, intelligent geographic information system. Afterwards, Frank [4] has studied the basic ontology in spatial database, pointing out that the utilization of ontology in geography helps to avoid the inconsistency of concepts in traditional systems. Harvey [5] introduces methods of semantics ontology in the design of a geographic information system and points out that it is efficient in overcoming the semantic differences in geography; V is s e r et al. [6] provide an overview of formalized ontology in geographic information and its application in processing the information, in addition to comparing the extent of formalization of different ontology languages and briefly describes an agency-based framework for an intelligent information retrieval. In the context of gradual application of ontology to various fields of studies on geographic information, the concept of Geo-Ontology was first put forward at the Geographic Information Science Conference, held in the UK in September 2002. This conference defines 
geographic ontology as study of the detailed connotation and relations on different levels and application fields of the concepts of geographic spatial information, and provides a semantic identity for the concept.

In the recent decade, after the concept of geographic ontology was put forward, geographic ontology was reliant upon the continuous development of information science. Therefore, there was little difference between Geographic Information Ontology and Geo-Ontology. Ontology-based studies of geographic information have been attracting wide academic interest. A number of scholars and academic societies have produced studies with positive results. For instance, Dr. Cui Wei [7] studies semantic integration and interoperability in an ontologybased geographic information system. Abdelmoty et al. [8] cover the key role of geographic ontology in the development of a spatial sensor search engine and evaluates the expression of geographic ontology and its inferential capability through the Geography Markup Language (GML) and Web Ontology Language (OWL). Dr Huang Maojun proposes an exact meaning of ontology in the geographic information science and explains the significance of building geographic ontology. In [9] $\mathrm{He}$ focuses his study on the ontology-based improvement of mapping services; Hes s and I o chpe [10] put forwards a set of methods to construct ontology using geographic ontology theory and reverse engineering, which is aimed at addressing the homogeneity of semantic granularity in ontology-based description. With the purpose of tackling the structural differences in data in the process of data integration and semantic nonhomogeneity, Khelifa and Mimoun [11] study the similarity in different ontologies and use the measure of the degree of similarity in the output of semantics to reflect the semantic relations in two geographic concepts. On ch a ga et al. [12] construct a set of systems and a framework which is used to evaluate the geographic information services. Liu Wei [13] makes attempts to build a geographic ontology database that corresponds to the description of spatial data services, and successfully realizes the discovery and integration of spatial data services. Ge W e n [14] makes studies on ontology-based semantics and the supporting rules of geographic information services discovery; he analyzes the norms, methods and tools of building geographic information ontology in addition to the composition of logics and methods of integration in geographic information ontology.

In general, some recent researches on geographic information ontology still stay at the theoretical stage. The research field focuses on several aspects, including the formal representation and modelling of geographic ontology, the semantic integration of heterogeneous geographic data, and the discovery and retrieval of the geographic data. These types of researches mainly concentrate on the semantic features of the geographical attributes. The purpose is to establish a hierarchy relationship between the geographic semantics, which clarifies various semantic relations, such as inclusion, equality, intersection and disjoint. It lays the foundation for the integration, retrieval and interoperation of geographic information.

Another trend of the researches is directed towards applications in a specific geographic information system environment, which aims at constructing geographic information ontology in specific areas with the use of specific semantic technology. 
It provides solutions to the problems in traditional construction, classification and retrieval of the geographic entities. This kind of researches is also one of the potential directions to break-through the current research of geographic information ontology. Its basic methodology is the migration of the application of ontology in information science towards geographic information science.

\section{Semantization of the Web services and geographic information services ontology}

Since there is a variety of fields in geosciences and complex classifications on different levels of granularity, the current researches of application only deal with the semantic expression of the geographic information in a specific field or ontology-based retrieval in a particular application environment. In recent years, the release and sharing of various types of information have adopted relevant standards based on the continuous improvement of Web services technology. Researches on semantization and ontology which were built upon the standard Web services technology, are developing correspondingly. Researches on ontology based on Web geographic information services will be an important research field in the subject of Geographic Information Ontology.

\subsection{Semantization of the Web services}

A traditional Web service could be seen as Internet application, with low coupling and independent from the platform. Through an open XML markup language, the service describes, releases, discovers, coordinates and allocates these applications. It is used for developing interoperable applications in heterogeneous Internet environments. The construction and release of the current standard Web services are connected with the construction of XML-based Web Services Description Language (WSDL). The current service conducts the registration and release through Universal Description Discovery and Integration (UDDI), for the purpose of unifying the automation of the applications in Internet. The current standard has advantages in using a grammatically accurate language to describe the dispatch of services. It has a high degree of automation in the expected dispatch of modes, the order and the type of parameters used for a dispatch of the operations. In addition, the technology of Web services retrieval based on key words is already mature. The drawback of the service which is based on WSDL norms is the lack of consideration of the semantics in the description of a service. It results in loss of information regarding the meaning of a service, and a relatively low rate of recall and precision. Facing the need of semantization of Web services and the problems arising from the practical applications, a large number of studies has been dedicated to this area in the past decade. Maximilien and Singh [15] have proposed a dynamic framework for Web Services retrieval based on the technology of ontology. B en saber and Malki [16] have proposed the use of a model driven approach to construct semantics in Web services. In [17] Dr Zeng Cheng constructs an interoperable framework for the registration and management of the Web service 
[16] in his dissertation which provides a summary of the interoperability of two types of semantics in Web services [17]. In [18] Dr Wang Hui puts forward a method for automatic labeling of the Web services and proves that it could remarkably enhance the accuracy of labeling and further proposes an ontologybased method of mining the relationships in Web services.

The relevant studies above presented show that the current methodology of studying the semantization of Web services is built upon the existing information technology and the realization of a series of automatic operations through the discovery of semantics in Web services in Internet environment. The methodology can be divided into two different approaches. The first approach is to add semantic descriptions to the current grammatically-based Web service description, labeling the semantics of the Web services described in documents through the upward compatibility of WSDL. Generally, this can be realized through standardized and extendible WSDL elements and attributes. The representative of this slight improvement in the semantic approach is the project norm of WSDL-S (WSDL-Semantics), which is made by W3C. The second approach is to build an ontology framework of Web services from the perspective of the current technology of semantic ontology, as shown by the Semantic Web Services Framework SWSF released by W3C. The framework combines Semantic Web Services Language SWSL with Semantic Web Services Ontology SWSO, providing a process-oriented model. Apart from the above mentioned approaches, the OWL-S OWL-based Web service ontology is proposed on the basis of extension of the standard of Web Ontology Language OWL. It is used for semantic information and related operations of describing the Web services and has been accepted by many overseas researchers [19-22]. In the light of continuity of the technology, the first approach of semantization of the Web services is easier and faster to realize. However, given the diversification of the specific geographic information services and the modularity of the system, it is more reasonable to build independent databases for semantic ontology of the Web services.

\subsection{Geographic information services ontology}

In another aspect, the purpose of the construction of a geographic information system is to organically integrate all data sources (including spatial and non-spatial data), which are geographically distributed, self-governed and heterogeneous. The system enables the customers to gain transparent access to any spatial data, and the functions and techniques required to handle this data. It provides the most useful information to the end-users in a quick manner through two major forms: data service and function service. Compared to the Web services in information science, the geographic information service has also independent standardization systems. The main system is put forward by TC 211 affiliated with ISO (International Organization for Standardization) in ISO19119, defining the concept, classification and service framework of a geographic information service. It divides the various kinds of services in a geographic information domain into six categories: human interaction services, model/information management services, workflow/task services, processing services, communication services, and system management 
services. The Open Geospatial Consortium OGC divides the categories of geographic information services more specifically, it puts forward as well a series of abstract standards and regulations, and defines the framework and interoperability protocol for OpenGIS Web Service (OWS). According to the existing realization and application of the above-mentioned geographic information service standards and protocols, the main information technologies that they rely on are still UDDI and WSDL in the current Web service environment. That is to say, the mainstream information presentation of the geographic information service is still realized by the syntactic structures in regulations. Therefore, the difficulties of heterogeneity in the information format, semantic multiplicity and lack of information relations are still general among the realization and integration of an enormous amount of geographic information services. In order to make the servers distributed in the network organize the services more automatically and efficiently, the factor of service semantics must be taken into consideration.

We opine that with the development of Web services semantization, we could introduce the concept and technology of Web Services Ontology into geographic information services, so that we can make use of the rich semantics and extensive relations of ontology, therefore solve the above problems and improve the quality of the geographic information services in the future. Web service ontology is the basis for semantization of the Web services. Introducing ontology into the service matching process, unlike the simple keyword-based matching algorithm, will certainly significantly improve the service matching precision and recall level. The service ontology, achieving automation of the service, provides also the semantic model shared by the applications, so that this enables the service to be interoperated at a semantic level. For example, in the study of the existing geographic information ontology system, Kolas, Hebeler and Dean [23] considered geographic information services ontology as one of the five main types of geographic ontologies under the geospatial semantic Web, and they believed its purpose is to make the knowledge base identify and implement the registered geo-spatial information service. Besides, in the specific area of geographic information services applications, such as in references [24-26], the issue of how to apply the service ontology technology in a simple geographic service, such as discovery and retrieval has been proposed. However, there is no systematic summary of the geographic information service ontology in terms of its concept, characteristics and applications. As it can be seen, although there are less application cases of the existing ontology technology, the basic application model of geographic information service ontology has made some progress, and it has a certain value and prospects in the geographic information services field. However, it is worth studying how to target the characteristics of the geographic information service itself, build its own Web service ontology framework and conduct a rational application.

In summary, as shown in Fig. 1, starting from the two perspectives of ontology and the service, we believe that geographic information service ontology is the combination product derived from the geographic information ontology and semantics in Web services, and it is an important component of the geographic 
information service ontology system. In this paper we define the geographic information service ontology as "Based on the specific geographic information service system and syntax description, making specification for the detailed semantic characteristic of the geographic information and its interoperation.” The direct application objects of the geographic information service ontology are the enormous amount of the geographic information services which are within a specific range, related to each other, and require semantic description. The aim of constructing the ontology is to complement the necessary semantic information to the existing geographic information services, so that the services can receive a more accurate description, thus to address issues caused by the semantic ambiguity and understanding ambiguity during the geographic information services integration application process, and further enable the rational and efficient use of the geographic information service.

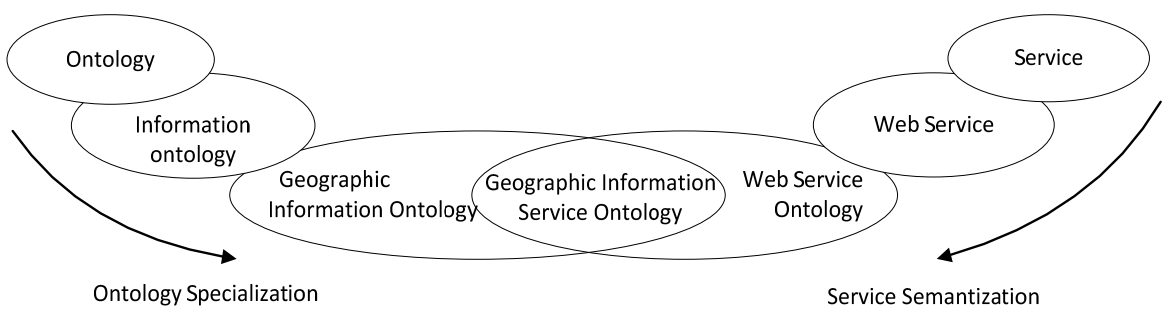

Fig. 1. Development of the geographic information ontology

4. Geographic information service ontology construction process and application framework

From the concept, application objects and construction purpose of the geographic information services ontology summarized above, we can see that the geographic information services ontology mainly serve in a certain scale, the integrated application environment of the geographic information services, and thus enhance the semantic characteristics of the service ontology. For example, Changzhou Hightech Zone, based on the SOA framework, has initially established a regional level spatial information services and an information sharing service platform (Fig. 2, left). In order to improve the precision in service inquiries and service combination utilization, the description can be adjusted according to the actual needs of the platform in the project (Fig. 2, right). Based on the characteristics of the geographic information services, a reasonable geographic information service ontology database can be established and applied in the sub-modules of the platform backstage.

According to the requirements, geographic information service ontology support can be added to extend the following modules: the ServiceProvider module can take advantage of the standardized semantic description of the geographic service, and using the additional geographic service ontology extraction tool in the ProvideService module to conduct semantic registry for the existing geographic information services; the RequestService module provides a variety of geographic 
information service ontology database interfaces to the ServiceUser module, which will improve the query efficiency and accuracy of the geographic information service, and enable the ServiceUser to logically select various types of geographic information services and construct the OperateServiceProcess module. Overall, all the relevant requirements can be categorized into two questions: how to construct the geographic information service ontology and how to utilize it.
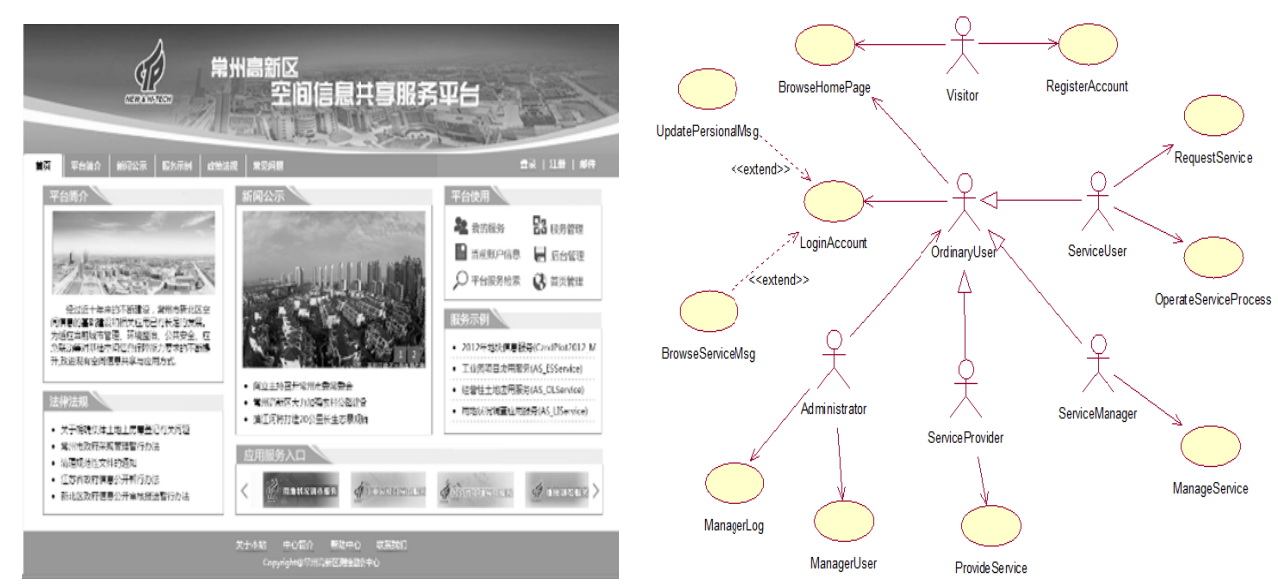

Fig. 2. GIS Platform and its requirements specification

\subsection{Geographic information service ontology construction process}

To build the geographic information services ontology is to solve the problem of how to generate geographic information service ontology. According to the research and analysis on the semantization of a Web service and the characteristics of the geographic information services in the second part of this paper, we think that in order to build the ontology in a mature geographic information service system, it is more likely to adopt the way of building independent ontology databases. In this way, not only the continuity and availability of the existing geographic information services can be guaranteed, but it can also realize low coupling of the additional semantic modules and existing system modules, therefore to facilitate the project implementation. In addition, the construction of a new geographic information service integrated application can also adopt the ontology database construction approach, and then conduct further modularization. To be specific, based on the actual situation, the process can be carried out in three steps as follows.

The first one is to determine the necessary concept of geographic information services, which can be determined based on the actual situation, using ISO19119 or OWS subsets to summarize or customize the geographic information service hierarchy structure at all levels according to the actual needs, characteristics of the field, and features of the software in use. For example, in a regional level platform environment like Changzhou High-tech Zone, the structure of a geographic information service can be defined as: 
a) Map atomic services, a map service that is published directly on ArcGIS Server. The services can be divided into two major categories - basic space and thematic space (Fig. 4, left).

b) Map extended services, named to the beginning "ES_", based on two types of atoms services. Through the standard Web services of GP or Java ADF extension development, various types of a service can be supported, such as the service process, application service, and other platform-related attribute information services which are provided by some specific business sectors.

c) Geographic information application service, named to the beginning "AS_", is a system-level application developed for specific geographical information services. It has a separate entrance page for users to directly log in, use and share unified authentication of the platform, and meanwhile it also provides a standard Web service interface for other services (Fig. 4, right).

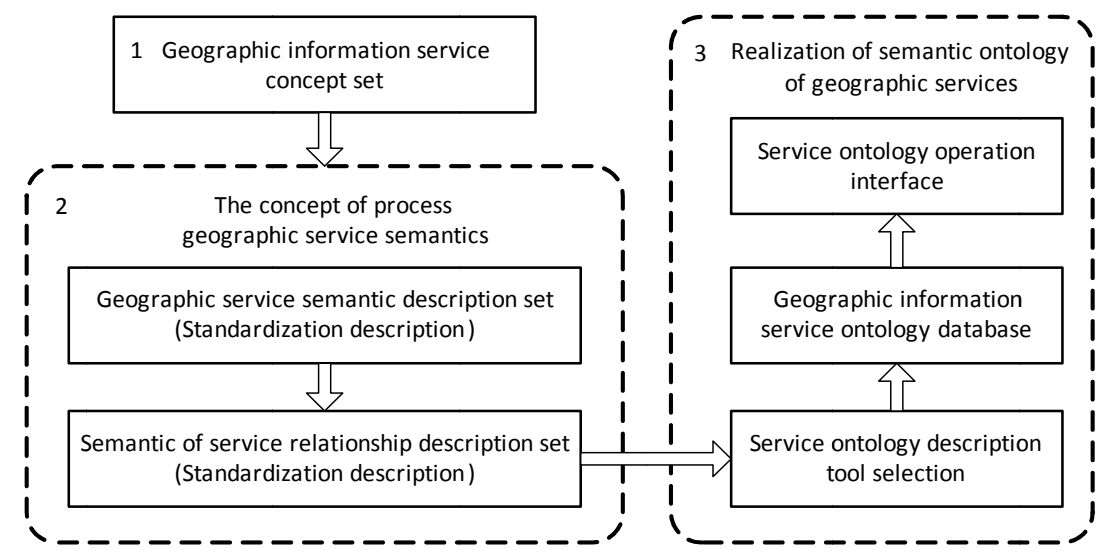

Fig. 3. Geographic information service ontology construction process
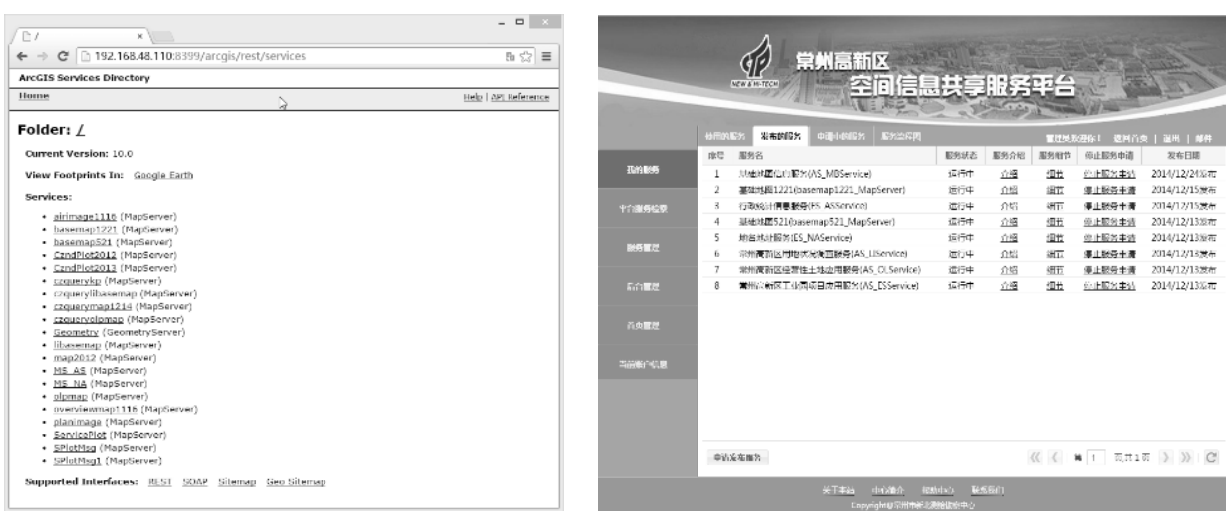

Fig. 4. Atomic service, extended service and application service under a GIS platform

The second step is to conduct semantic description of the geographic information services and inter-service relations, and provide standardized text description based on the basic geographic information standards and related specifications in the actual application. In addition, if the practical application 
requires combination of the services, or high-quality service matching, then the called dependencies between all levels of the service and various services must be considered, thus to statute the inter-service relations semantic description collection, and even to establish GIS semantic logic axioms for the subsequent use of geographic information spatial reasoning.

The final step is the realization of the specific geographic service ontologies, including the selection of the ontology description tools, the construction form of the geographic information ontology databases (to extract the ontology automatically or manually, store the data using a database or a file storage), and the organizational design of the geographic information service ontology interface. This process can be achieved using some open source-based API (such as OWL-S API) to encode and automatically obtain the data, or manually process it by using the graphical tools provided to obtain a specific geographic service ontology structure. For example, Fig. 6 shows a GIS ontology using an ontology editing tool directly constructed, based on OWL-S and a simple file storage structure.
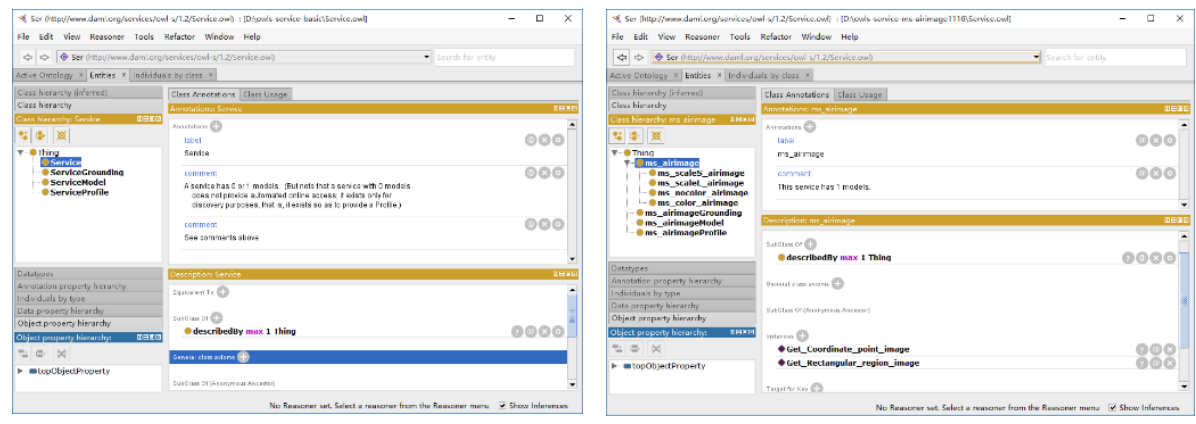

Fig. 5. A file-based geographic information service ontology artifact

\subsection{Geographic information service ontology application framework}

The application framework of GIS ontology, built on the basis of GIS ontology database, is in line with the objective practice of information engineering technologies. The existing GIS ontology and semantic integration of the services can be considered the core of its construction, while the whole framework must be in line with the SOA ideological framework. That is to say, through a stable network transmission, under the relevant policies and regulations, and the existing standards and information security constraints, to construct and realize multisource, mass-based information resources services and related management, allowing it achieve data interoperability among heterogeneous platforms by providing Web services standardized second development interface.

As shown in Fig. 6, based on an existing geographic information sharing service platform, we can use the simple three-layer structure elements to construct and expand the GIS ontology application framework. The bottom layer is the GIS ontology library which is constructed in Section 3.1, the middle layer is the service ontology file user interface which is based on the ontology library, and the top layer is the Enterprise Service Bus (ESB) interface module which is constructed based on calling these interfaces and is consistent with the legacy system architecture. The 
technical implementation of this module has to be based on the existing technology architecture. In order to facilitate unified management, this module can also be defined as a new service component registered on the ESB and providing functions to other modules to use. For instance, Fig. 7 illustrates, under a GIS platform, the process carried out through the module, including service selection, service matching, service forming and execution.

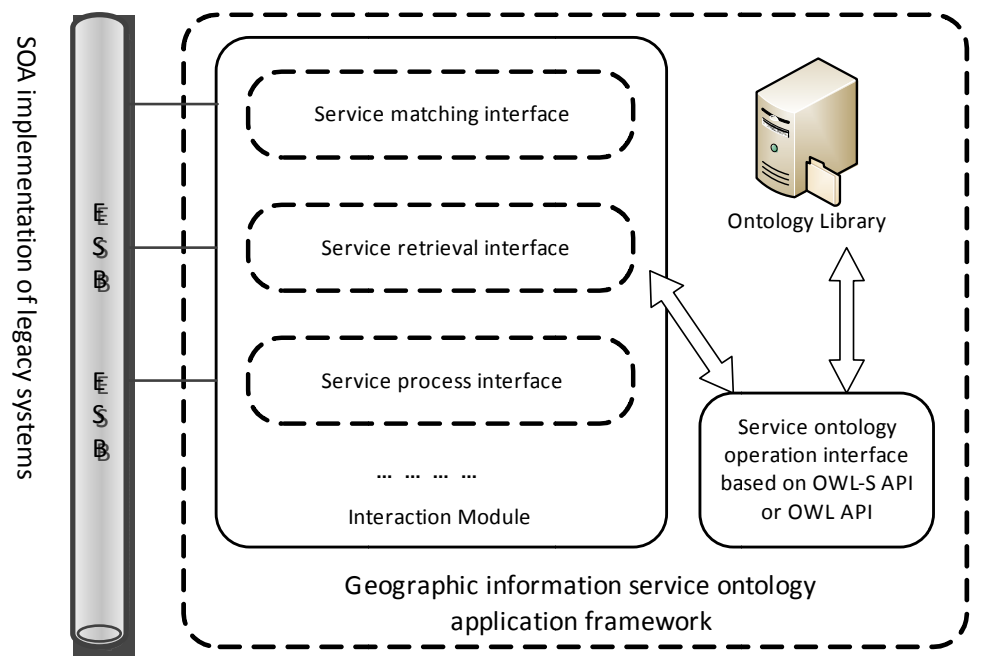

Fig. 6. Geographic information service ontology application framework
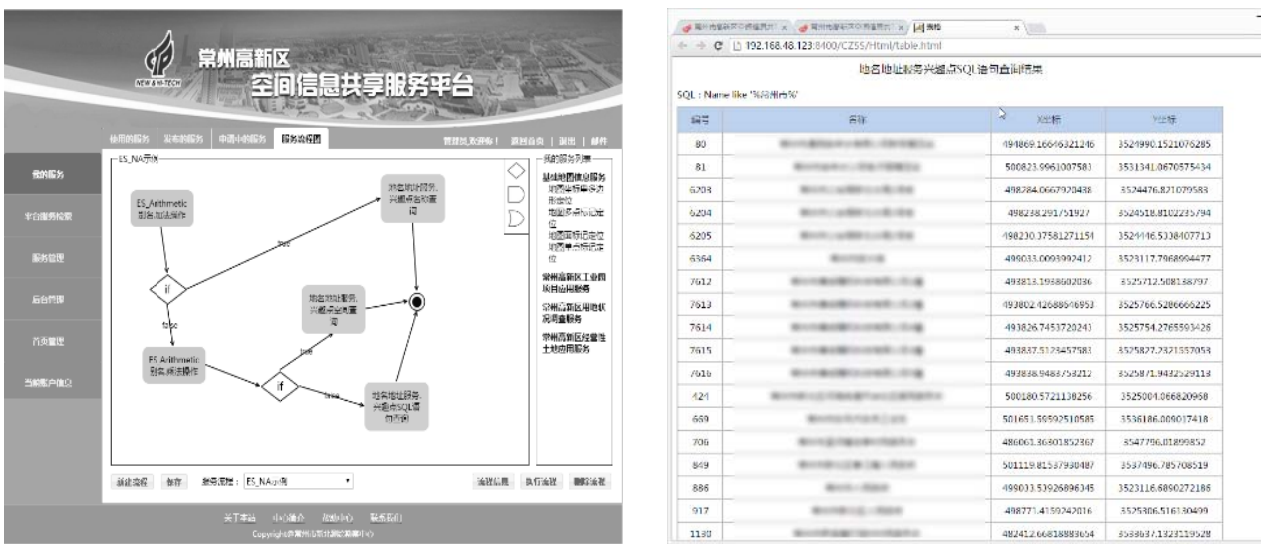

Fig. 7. GIS process and results

\section{Conclusion}

Through the above review of GIS ontology and its application development, we can make the following conclusion. With the current network and related information technology, in order to describe GIS under heterogeneous environment the 
traditional pure grammatical structure-based method must evolve to an integration approach which is more bound to the syntax and additional semantics, so that we can obtain a more accurate service operation under the desired Web environment, thus improving the quality of the geographic information services. In this connection, the geographical information service ontology related research will become an important research direction of geographic information ontology. Finally, by a case study, this paper has proposed and implemented a feasible approach to construct GIS ontology and its application framework, and demonstrated the availability and feasibility of GIS ontology in practice.

Acknowledgements: The research work was supported by the National Cultural Information Resources Sharing Project in the National Science and Technology Pillar Program of China (Grant No 2012BAH01F02) and WuHan Talent Program.

\section{References}

1. Gruber, T. R. A Translation Approach to Portable Ontology Specifications. - Knowledge Acquisition, Vol. 5, 1993, pp. 199-220.

2. N u n e s, J. Geographic Space as a Set of Concrete Geographical Entities. D. Mark, A. Frank, Eds. Cognitive and Linguistic Aspects of Geographic Space. Netherlands, Springer 1991, pp. 9-33.

3. E g e $n$ h o f e r, M., D. M a r k. Naive Geography. A. Frank, W. Kuhn, Eds. Spatial Information Theory A Theoretical Basis for GIS. Berlin, Heidelberg, Springer, 1995, pp. 1-15.

4. Fra n k, A. Spatial Ontology: A Geographical Information Point of View. O. Stock, Ed. Spatial and Temporal Reasoning. Netherlands, Springer, 1997, pp. 135-53.

5. H a rve y, F. Designing for Interoperability: Overcoming Semantic Differences. M. Goodchild, M. Egenhofer, R. Fegeas et al., Eds. Interoperating Geographic Information Systems. Springer US. 1999, pp. 85-97.

6. Vis s e r, U., H. S tuckenschmidt, G. Schuster et al. Ontologies for Geographic Information Processing. - Computers \& Geosciences, Vol. 28, 2002, No 1, pp. 103-117.

7. We i, C. Using Ontology to Achieve the Semantic Integration and Interoperation of the Geography Information System. Wuhan University, 2004.

8. A bdelmoty, A. I., P. D. S mart, C. B. Jon es et al. A Critical Evaluation of Ontology Languages for Geographic Information Retrieval on the Internet. - Journal of Visual Languages \& Computing, Vol. 16, 2005, No 4, pp. 331-58.

9. M a o j u n, H. Study on Formal Representation of Geographic Ontology and Its Application in Map Services. Wuhan University, 2005.

10. H e s s, G. N., C. I o c h p e. Geo-Ontology Enrichment through Reverse Engineering. - In: Proc. of 16th ACM SIGSPATIAL International Conference on Advances in Geographic Information Systems, ACM GIS 2008, 5-7 November 2008, Irvine, CA, United States, F, 2008. Association for Computing Machinery.

11. Khelifa, D., M. Mimoun. Ontology Based Semantic Integration of Heterogeneous Geographical Information Systems. - In: Proc. of 2008 3rd International Conference on Information and Communication Technologies: From Theory to Applications, ICTTA, 7-11 April 2008, Damascus, Syria, F, 2008. Inst. of Elec. and Elec. Eng. Computer Society.

12. Onchaga, R., I. Widya, J. Morales et al. An Ontology Framework for Quality of Geographical Information Services. - In: Proc. of 16th ACM SIGSPATIAL International Conference on Advances in Geographic Information Systems, ACM GIS 2008, 5-7 November 2008, Irvine, CA, United states, F, 2008. Association for Computing Machinery.

13. W e i, L. Spatial Data Service Discovery and Integration Based on Geo-Ontology. China Mining University, 2010.

14. W e n, G. Research on the Method of Geographic Information Service Discovery. The PLA Information Engineering University, 2012. 
15. Maximilie n, E. M., M. P. S ingh. A Framework and Ontology for Dynamic Web Services Selection. - IEEE Internet Comput., Vol. 8, 2004, No 5, pp. 84-93.

16. B ensaber, D. A., M. Malki. Development of Semantic Web Services: Model Driven Approach. - In: Proc. of 8th International Conference on New Technologies in Distributed Systems. Lyon, France, ACM, 2008, pp. 1-11.

17. C h e n g, Z. Mechanism and Methodology of Web Service Registry and Repository for Semantic Interoperability. Wuhan University, 2010.

18. $\mathrm{Hu}$ i, W. Construction and Application of Web Service Infrastructure for Internet. Tianjin University, 2010.

19. M a r t i n, D., M. B u r s te i n, D. M c d e r m o t t et al. Bringing Semantics to Web Services with OWL-S. -World Wide Web, Vol. 10, 2007, No 3, pp. 243-277.

20. K i m, I. W., K. H. L e e. A Model-Driven Approach for Describing Semantic Web Services: From UML to OWL-S. - IEEE Trans. Syst. Man Cybern. Part C - Appl. Rev., Vol. 39, 2009, No 6, pp. 637-646.

21. Me ditsko s, G., N. B a s siliades. A Combinatory Framework of Web 2.0 Mashup Tools, OWL-S and UDDI. - Expert Syst. Appl., Vol. 38, 2011, No 6, pp. 6657-6668.

22. Farrag, T. A., A. I. S a le h, H. A. A li. Toward SWSs Discovery: Mapping from WSDL to OWL-S Based on Ontology Search and Standardization Engine. - IEEE Trans Knowl. Data Eng., Vol. 25, 2013, No 5, pp. 1135-1147.

23. K ol a s, D., J. H e b e le r, M. D e a n. Geospatial Semantic Web: Architecture of Ontologies. Lect. Notes Comput. Sci., Vol. 3799, 2005, pp. 183-194.

24. Li ang, H., H. Z ongyi, X. Jian et al. Geography Information Web Service Semantic Description and Automatic Discovery Based on Web Service and OWL-S. - In: Proc. of 1st International Workshop on Database Technology and Applications, 2009, F, 25-26 April 2009.

25. Peng, S.-Y., W.-F. Ma, K. Y ang et al. A Practical Study on Geographic Semantic Web Services Based on OWL-S. - In: Proc. of International Conference on Computing, Control and Industrial Engineering (CCIE), 2010, F, 5-6 June 2010.

26. J i a n g, L., Y. J i a n g. Semantic Web Enabled Intelligent Geospatial Web Services; Proceedings of the Practical Applications of Intelligent Systems. - In: Proc. of 6th International Conference on Intelligent Systems and Knowledge Engineering, Shanghai, China, December 2011 (ISKE'2011), Tiergartenstrasse 17, Heidelberg, D-69121, Germany, F, 2011. Springer Verlag. 